\title{
Diagnostic Value of the Reflux Disease Questionnaire in General Practice
}

\author{
M.C. Aanen ${ }^{a}$ M.E. Numans ${ }^{\text {b }}$ B.L.A.M. Weusten ${ }^{\text {c }}$ A.J.P.M. Smout ${ }^{a}$ \\ ${ }^{a}$ Gastrointestinal Research Unit, b Julius Center for Health Sciences and Primary Care, \\ University Medical Center Utrecht, Utrecht, and ' Department of Gastroenterology, Sint Antonius Hospital, \\ Nieuwegein, The Netherlands
}

\section{Key Words}

Reflux disease questionnaire $\cdot$ Proton pump inhibitors •

Esomeprazole $\cdot$ Gastro-esophageal reflux disease •

Primary care

\begin{abstract}
Objectives: This study determined the diagnostic and therapeutic response of the Reflux Disease Questionnaire (RDQ) using the symptom association probability (SAP) as reference. In addition, the RDQ's construct validity and its relationship to quality of life (QOL) were ascertained. Methods: Seventy-four patients with GORD symptoms (age 51 years (22-78); $\$ 62 \%)$ derived from primary care completed the $\mathrm{RDQ}, \mathrm{GSRS}$ and QOLRAD before and after a 2 weeks' course of esomeprazole $40 \mathrm{mg}$ daily. The SAP was determined by a 24-hour $\mathrm{pH}$ recording before PPI treatment. The diagnostic abilities of the RDQ (total and 4 dimensions scores) were assessed with the area under the curve (AUC) of a receiver operating curve. RDQ scores before and after PPI treatment were compared with Wilcoxon tests. Multiple linear regressions assessed the RDQ's construct validity (GSRS) and relationship to QOL (QOLRAD). Results: The AUCs were low for all RDQ dimensions (AUC < 0.6). In the SAP-positive patients all RDQ dimensions improved $(p<0.0001)$ while the scores of the SAP negatives did not (heartburn $p<0.01 ;$ GORD and total score $p<0.05$; regurgitation and dyspepsia n.s.). The RDQ was related to the total and reflux GSRS dimensions while the food and drink QOL dimension was linearly associ-
\end{abstract}

ated with the RDQ. Conclusions: The RDQ is a valid and reliable questionnaire with excellent construct validity and a good relationship to QOL. The diagnostic value of the RDQ in primary care is limited, but combination with an additional PPI treatment course might improve the RDQ's ability to discriminate GORD patients according to their SAP outcome.

Copyright $\odot 2006$ S. Karger AG, Basel

\section{Introduction}

Gastro-esophageal reflux disease (GORD) is common in the western population [1]. Most GORD patients are diagnosed and treated in the primary care setting. Despite the fact that the disease itself is benign since the prevalence of complications and severe GORD-related morbidity is low, GORD can severely reduce a patient's quality of life $[2,3]$. This is why GORD treatment is usually started empirically in an early stage. Following treatment start, diagnostic procedures are postponed and a favorable response to proton pump inhibitors (PPI) is widely accepted to validate the diagnosis [4]. However, the increase in long-term use of PPIs without a proper diagnosis has urged researchers to look for other means to improve GORD diagnostics. One of the tools that might help the general practitioner (GP) is a validated GORD questionnaire to support diagnostic accuracy. It is likely that the use of a questionnaire would reduce the

\section{KARGER}

Fax +4161306 1234

E-Mail karger@karger.ch

www.karger.com (c) 2006 S. Karger AG, Basel

0012-2823/06/0744-0162\$23.50/0

Accessible online at:

www.karger.com/dig
M.C. Aanen, MD

Gastrointestinal Research Unit

University Medical Center Utrecht, PO Box 85500

NL-3508 GA Utrecht (The Netherlands)

Tel. +31 30250 9811, Fax +31 30250 5533, E-Mail m.aanen@umcutrecht.nl 
inter-observer variability in comparison with clinical history taking. Besides aiding in the diagnosis of GORD, a questionnaire would also enable the physician to quantify therapeutic response.

The Reflux Disease Questionnaire (RDQ) is a promising new questionnaire that was specially designed to be used in the primary care setting [5]. Extensive research has found this questionnaire to be reliable, valid, responsive and above all practical [5-7]. Furthermore, the RDQ outcome seems to correlate well with quality of life $[6,8]$. However, data on its diagnostic validity is still lacking [7].

This validation should be performed in a population that represents patients in whom primary care physicians consider the diagnosis of GORD and in this population the most relevant diagnostic reference test for GP patients should be used, which, in our opinion, is a measure of the symptom-reflux association, such as the symptom association probability (SAP) [9]. The SAP objectively determines with a Fisher exact test whether symptoms are due to reflux events taking all symptom episodes and reflux events into account.

The aim of this study was therefore to assess the diagnostic and therapeutic response of the RDQ questionnaire using the SAP outcome as determined by 24 -hour $\mathrm{pH}$ recording as reference standard in a primary care population. The secondary aim was to ascertain the construct validity of the questionnaire and to specify the RDQ's relationship with quality of life.

\section{Methods}

\section{Patients}

Seventy-four patients (mean age 51 years (22-78); 62\% male) who completed a 24 -hour $\mathrm{pH}$ recording and exhibited symptoms during this recording were analyzed in this study. These patients were recruited directly during a GP consultation (34\%) or indirectly by advertisement in a local newspaper (66\%). In case of recruitment by advertisement the patient's GP was consulted before inclusion. All patients had symptoms suggestive of reflux disease (i.e. heartburn, regurgitation, acid taste, burning sensation in the epigastric region, epigastric pain and chest pain) for at least twice a week for the past 3 months. The subjects had not used an acidsuppressant drug for at least 4 weeks before entry. Furthermore, none of the subjects had undergone gastrointestinal surgery.

Informed written consent was obtained before the start of the study and the protocol was approved by the Medical Ethics Committee of the University Medical Center, Utrecht.

\section{Study Protocol}

All patients with symptoms suggestive of GORD were asked to fill in the RDQ. In order to assess the RDQ's construct validity and the RDQ's assessment of quality of life, the Gastrointestinal
Rating Scale (GSRS) and Quality of Life in Reflux and Dyspepsia (QOLRAD) questionnaires were simultaneously completed with the RDQ 1 week before a 24 -hour $\mathrm{pH}$ recording $[5,10,11]$. After the $\mathrm{pH}$ recording patients used $40 \mathrm{mg}$ esomeprazole daily during 2 weeks. After these 2 weeks the patients completed the three questionnaires again. The SAP was determined after the $\mathrm{pH}$ recording but the patients and their physician were kept oblivious of the results until the protocol was completed.

\section{4-Hour pH Monitoring}

The 24-hour $\mathrm{pH}$ recording was performed after identification of the lower oesophageal sphincter (LOS) with manometry. The manometric recording was performed with a 10-channel silicone rubber catheter with a reversed sleeve sensor (DentSleeve International Ltd., Mississauga, Ont., Canada) which was perfused at a rate of $0.2 \mathrm{ml} / \mathrm{min}$ with degassed water, using hydraulic flow restrictors (DentSleeve International Ltd, Mississauga, Ont., Canada). The pressures were recorded with external pressure transducers (Abbott, Sligo, Ireland). The 24-hour $\mathrm{pH}$ recording was performed with a glass $\mathrm{pH}$ catheter with in-built reference electrode (Ingold, Urdorf, Switzerland) that was transnasally placed $5 \mathrm{~cm}$ above the LOS. The $\mathrm{pH}$ catheter was calibrated with 3.2 and $7.4 \mathrm{pH}$ buffers solutions. The $\mathrm{pH}$ catheter was then attached to a digital datalogger (MMS, Enschede, the Netherlands) which used a sampling frequency of $2 \mathrm{~Hz}$. All patients were instructed to record their symptoms by pressing the event marker button on the datalogger and at the same time specifying the symptom in a diary card. In the diary card also the times of consumption of meals and beverages and the recumbent time were noted. Patients were instructed to restrict their intake to 3 meals and 3 drinks during the $24 \mathrm{~h}$ at standardized times. Meals and drinks had to be consumed within 30 and $15 \mathrm{~min}$, respectively. Patients were encouraged to maintain their normal daily activities during the 24-hour $\mathrm{pH}$ study.

After the 24-hour recording period the data from the datalogger was transferred to a personal computer.

\section{Analysis of 24-Hour pH Data}

The 24-hour $\mathrm{pH}$ data were analyzed automatically (MMS, Enschede, the Netherlands), excluding all eating and drinking periods. The SAP is calculated by dividing 24 -hour $\mathrm{pH}$ data into consecutive 2-min segments. For each of these 2-min segments, it is determined whether reflux occurred, providing the total number of two-minute segments with (total $\mathrm{R}+$ ) and without (total R-) reflux. Then, for each symptom episode, it is determined whether reflux did $(\mathrm{S}+\mathrm{R}+)$ or did not $(\mathrm{S}+\mathrm{R}-)$ occur in the preceding twominute period. Subtraction of $\mathrm{S}+\mathrm{R}+$ from total $\mathrm{R}+$ results in $\mathrm{S}-\mathrm{R}+$ and subtraction of $\mathrm{S}+\mathrm{R}-$ from total $\mathrm{R}$ - results in $\mathrm{S}-\mathrm{R}-$. A $2 \times 2$ contingency table is then constructed in which the number of 2min segments with and without symptoms and with and without reflux are tabulated. Fisher's exact test is used to calculate the probability ( $p$ ) that the observed distribution could have been brought about by chance. The SAP is calculated as $(1-p) \times 100 \%$. By statistical convention, the SAP is considered positive if it exceeds $95 \%[9,12]$.

\section{Analysis of Questionnaires}

The English version of the RDQ comprises 12 questions assessing the frequency and severity of heartburn, acid regurgitation and dyspeptic complaints which are scored on a 5-point Lik- 
Fig. 1. Pretreatment (dark grey boxes) and posttreatment (light grey boxes) RDQ scores. Both before and after treatment the dimensions scores were similar. Treatment significantly reduced the RDQ scores for all dimensions. ${ }^{*}$ Pretreatment vs. posttreatment scores $\mathrm{p}<0.01$.

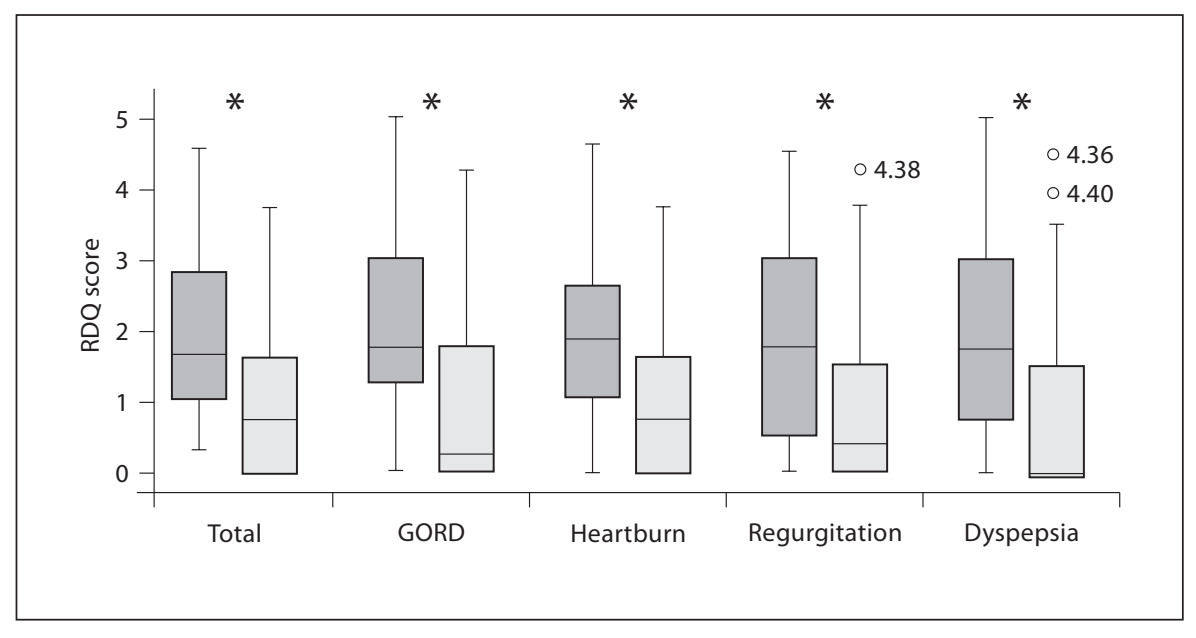

ert scale [5]. We used the Dutch version of RDQ which has been translated from English to Dutch and re-translated back for validity. The 12 items are combined into 3 dimensions: heartburn, regurgitation, dyspepsia. The mean of all three dimensions gives a total score ranging from 0 to 5 . The specific GORD dimension is determined by the mean of the dimensions heartburn and regurgitation.

The QOLRAD, a disease-specific quality-of-life questionnaire, covers five dimensions: emotional distress, sleep disturbance, problems with eating and drinking (food and drink problems), limitations in physical and social functioning and lack of vitality. The Dutch QOLRAD is similar to the original English version. Responses were rated on a 7-grade Likert scale. The lower the score, the more severe the impact was on daily functioning during the past week. The QOLRAD has been shown to be reliable and valid $[11,13]$.

The GSRS includes 15 items combined into five symptom clusters addressing to what extent different gastrointestinal symptoms were bothersome in the past week. The five symptom clusters depict reflux, abdominal pain, indigestion, diarrhea and constipation. The GSRS has a seven-graded Likert type scale where 1 represents absence of bothersome symptoms and 7 very bothersome symptoms. All questions were translated into Dutch. The GSRS is documented to be reliable and valid $[13,14]$.

\section{Statistics}

In order to determine significant differences a Mann-Whitney test was used for unpaired data and a Wilcoxon test for paired data. A p $<0.05$ was considered statistically significant. Data is presented in median and interquartiles (25-75th).

The ability of the RDQ to discriminate SAP-positive from SAP-negative patients was quantified by using the receiver operating curve (ROC) [15]. The area under the curve (AUC) denotes the discriminative power of a diagnostic model and can range from 0.5 (no discrimination, like flipping a coin) to 1.0 (perfect discrimination). A value of $0.7-0.8$ is considered to represent a reasonable diagnostic test and a value of $>0.8$ represents a good discriminative diagnostic test [16]. In case of a good discriminative AUC, an optimal cut-off point can be determined at the larg- est angle of the curve, most closely related to the left upper corner of the figure.

To compare the RDQ dimensions with the pretreatment quality of life outcome of the QOLRAD and to assess the construct validity with the GSRS a stepwise multiple regression was performed. The 5 RDQ dimensions were subsequently chosen as the dependent factor. In a multiple linear regression model, adjusted $\mathrm{R}$ square $\left(\operatorname{adj} \mathrm{R}^{2}\right)$ measure the proportion of the variation in the dependent variable accounted for by the explanatory variables thereby making adjustments for the number of explanatory variables inserted into the model. The adjusted $\mathrm{R}$ squares can take on any value between 0 and 1, with a value closer to 1 indicating that a greater proportion of variance is accounted for by the model. In this study all adjusted $\mathrm{R}$ squares are shown in percentages.

\section{Results}

Similar pretreatment scores were found for the five RDQ dimensions that were studied. Also posttreatment the symptom scores of the five RDQ dimensions were also similar. However, PPI treatment significantly reduced all RDQ symptom scores (fig. 1).

Seventy percent of the studied subjects had a positive SAP. Patients with and without a positive SAP had similar pretreatment RDQ scores (table 1). On PPI treatment, patients with a positive SAP appeared to have a greater improvement of their RDQ scores: only in subjects with a positive SAP all RDQ dimensions scores were significantly reduced (table 1 ). Treatment decreased the total score significantly more in SAP-positive than in SAPnegative patients $(\mathrm{p}<0.05)$.

The ROC analysis showed that all RDQ dimension scores had an AUC $<0.6$ with the SAP as reference stan- 


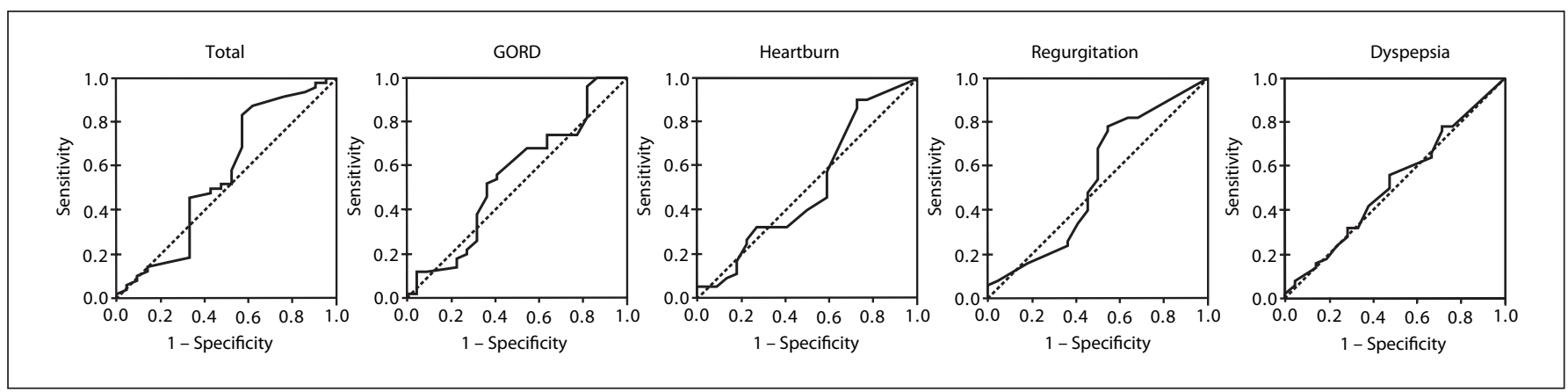

Fig. 2. ROC curves of the total RDQ scores and the 4 RDQ dimensions versus the SAP outcome. The sensitivity represents the true-positive fraction and 1 - specificity the false-positive fraction at different cut-off points. All dimensions scores followed the broken reference line, indicating that every improvement in false-positive rate is matched by a corresponding decline in the false-negative rate corresponding with areas under the curves below 0.6 (table 2). Therefore, no diagnostic cut-off point could be identified.

Table 1. Pre- and posttreatment scores for all RDQ dimensions for SAP-positive and SAP-negative patients

\begin{tabular}{|c|c|c|c|c|c|}
\hline \multirow[t]{2}{*}{ Dimension } & \multicolumn{2}{|c|}{ Pretreatment } & \multicolumn{2}{|c|}{ Posttreatment } & \multirow[t]{2}{*}{$\mathrm{p}$ value } \\
\hline & median & $25-75$ th & median & $25-75$ th & \\
\hline \multicolumn{6}{|l|}{ SAP-positive } \\
\hline Heartburn & 1.75 & $1.50-3.44$ & 0.00 & $0.00-1.50$ & $<0.0001$ \\
\hline Regurgitation & 1.75 & $0.94-2.81$ & 0.25 & $0.00-1.50$ & $<0.0001$ \\
\hline Dyspepsia & 1.88 & $0.94-3.06$ & 0.00 & $0.00-1.25$ & $<0.0001$ \\
\hline GORD & 2.13 & $1.00-2.63$ & 0.63 & $0.00-1.50$ & $<0.0001$ \\
\hline Total score & 1.83 & $1.33-2.88$ & 0.42 & $0.00-1.50$ & $<0.0001$ \\
\hline \multicolumn{6}{|l|}{ SAP-negative } \\
\hline Heartburn & 2.13 & $0.38-3.13$ & 0.50 & $0.00-2.31$ & $<0.01$ \\
\hline Regurgitation & 1.38 & $0.00-3.25$ & 0.75 & $0.00-2.50$ & n.s. \\
\hline Dyspepsia & 1.50 & $0.25-3.13$ & 0.75 & $0.00-2.75$ & n.s. \\
\hline GORD & 1.56 & $1.09-3.16$ & 1.25 & $0.00-1.78$ & $<0.05$ \\
\hline Total score & 1.67 & $0.88-3.04$ & 1.00 & $0.42-1.96$ & $<0.05$ \\
\hline
\end{tabular}

$\mathrm{p}$ values relate to differences between pre- and posttreatment scores.
Table 2. Area under the curve (AUC) for all RDQ dimensions found during ROC analysis against the SAP outcome as reference standards

\begin{tabular}{llllll}
\hline Indices & Dimensions & AUC & SEM & \multicolumn{2}{l}{$\begin{array}{l}\text { 95\% asymptotic } \\
\text { confidence interval }\end{array}$} \\
\cline { 3 - 6 } & & & & lower & upper \\
\hline SAP & heartburn & 0.51 & 0.08 & 0.36 & 0.66 \\
& regurgitation & 0.54 & 0.08 & 0.38 & 0.70 \\
& dyspepsia & 0.52 & 0.08 & 0.37 & 0.67 \\
& GORD & 0.55 & 0.08 & 0.40 & 0.70 \\
& total score & 0.56 & 0.08 & 0.40 & 0.73 \\
\hline
\end{tabular}

Reflux Disease Questionnaire in General Practice dard (table 2). The RDQ dimension scores from patients recruited by advertisement or by their GP showed similar AUC values both below 0.6 (data not shown). No RDQ cut-off value could be determined that would separate SAP-positive from SAP-negative patients (fig. 2).

The RDQ dimensions GORD and heartburn were found to be linearly related with the GSRS reflux score and they could be explained by the GSRS for 57\% and $29 \%$, respectively. Also regurgitation and the total RDQ score were positively related to GSRS reflux score (regurgitation adj $\mathrm{R}^{2} 40 \% \mathrm{p}<0.001$; total RDQ adj $\mathrm{R}^{2} 44 \% \mathrm{p}<$ $0.001)$. By adding the total score of bothersome gastroin- 
testinal symptoms the regression model improved (regurgitation: reflux and total $\operatorname{adj}^{2} 47 \% \mathrm{p}<0.05$; total RDQ: reflux and total adj $\mathrm{R}^{2} 60 \% \mathrm{p}=0.01$ ). The dyspepsia dimension was solely related to the total GSRS score $\left(\operatorname{adj} \mathrm{R}^{2} 34 \% \mathrm{p}<0.001\right)$.

Assessment of the relationship between the QOLRAD scores and the RDQ scores showed that the total RDQ score and the RDQ dimension GORD could both be explained for $40 \%$ and the RDQ dimension heartburn for $33 \%$ by the QOLRAD dimension food and drink problems (all 3 regressions $\mathrm{p}<0.001$ ). Regurgitation was also associated with problematic food and drink intake and the variance of this dimension could be explained for by $19 \%(\mathrm{p}<0.01)$. The dimension physical and social dysfunction accounted for an extra 5\% elucidation of regurgitation $(\mathrm{p}<0.05)$, but this was due to three outliers. After exclusion of these outliers regurgitation was only associated with food and drink problems ( $\left.\operatorname{adj} \mathrm{R}^{2} 17 \%, \mathrm{p}<0.001\right)$. The dyspepsia dimension did not relate to a specific QOLRAD domain but was linearly related to the total QOLRAD score $\left(\operatorname{adjR}^{2} 16 \%\right.$, $\mathrm{p}<0.001)$.

\section{Discussion}

The main findings of our study are: (1) The RDQ cannot be used to identify GORD patients as defined by a positive SAP outcome. The areas under curves were lower than 0.6 for all dimensions and subsequently no optimal cut-off value could be determined. (2) After PPI treatment all RDQ scores were significantly reduced in SAP-positive patients but not in SAP-negative patients and the reduction in total RDQ score after treatment was greater in SAP-positive than in SAP-negative patients. (3) The RDQ questionnaire is specific for reflux symptoms but not for other bothersome gastrointestinal symptoms as assessed by GSRS. (4) The RDQ relates to QOL, i.e. to the dimension food and drink problems.

The RDQ, assessing frequency and severity of reflux symptoms to facilitate the diagnosis of GORD in primary care, presently is the best-designed GORD-specific questionnaire, due to the fact that both expert opinions and patient's interview analyses were used in its development $[5,7]$. According to our study results, however, RDQ does not deliver the solution for misclassification problems in primary care. This finding needs further discussion. It has been shown that clinical history taking alone has a low specificity for diagnosing GORD and is riddled with a high interobserver variability, despite a high sen- sitivity [17]. Nowadays, most GPs have adopted the PPI test for diagnosing GORD, although this has also shown to yield a low specificity, despite a high sensitivity [17-19]. Due to the rebound effect of PPIs even more patients are false-positively labeled [20]. This leads to the conclusion that currently no adequate diagnostic test for GORD can be performed in primary care and thus, the vast majority of patients with suspected GORD symptoms have to be treated empirically. Subsequently, in most patients a diagnosis is never made, which may lead to a life-long treatment with a PPI without knowing whether this is the best treatment option.

Performing a more invasive and costly investigation in every patient is not possible in a primary care setting. Furthermore, due to the fact that no absolute gold standard exists, it is unclear which diagnostic test would be most useful. Endoscopy is irrelevant in a primary care population in which the majority of GORD patients do not have acid-induced oesophageal lesions $[3,17]$. We believe that the occurrences of GORD symptoms are caused by reflux events. Calculation of the SAP determines whether a true relationship exists between symptoms and reflux events and is therefore a more relevant procedure than only an assessment of the percentage of time with oesophageal $\mathrm{pH}$ below $4[9,12]$. For the evaluation of the diagnostic properties as well as the responsiveness to treatment of the RDQ questionnaire we have used the SAP outcome as the reference standard for presence of GORD. A questionnaire validated against the SAP that would demonstrate whether reflux symptoms of primary care patients are due to acidic reflux events would greatly improve GORD diagnosis in primary care [12].

Disappointingly however, the RDQ was unable to discriminate SAP-positive from SAP-negative patients, despite the fact that our study population was comprised of patients in whom a GP indeed might use a questionnaire and/or short-term PPI treatment to support diagnostic decisions $[18,21,22]$. Although our study population was 'GORD-enriched', this does not influence the characteristic test properties, i.e. sensitivity and specificity [23]. The observation that patients recruited from two different sources did not show different outcomes in AUC suggests that the performance of the RDQ is not due to issues related to patient selection.

Several studies have shown that the response to PPI treatment, when simply evaluated with symptom improvement or patient satisfaction scores, results in a large group of false-positively labeled patients $[18,19]$. It is possible that the additional use of the RDQ would enable 
physicians to improve the diagnostic test characteristic of the PPI test. Our data suggest that patients with a positive SAP had significantly better posttreatment RDQ scores than patients with a negative SAP while the reduction in total RDQ score was significantly greater in SAP-positive patients. However, these results only show differences between groups and may not be applicable to individual patients. Nevertheless, our results suggest that the RDQ could be used as an additional help in differentiating GORD from non-GORD in the relevant population of patients suspected of GORD in primary care, when shortterm empirical treatment with PPI is considered. Further research is needed to determine whether the response of the RDQ scores to PPI treatment can indeed be used diagnostically.

The RDQ has been shown to have excellent construct validity for GORD which we confirmed with the GSRS questionnaire [5, 7]. All reflux-specific RDQ domains were strongly related to the reflux dimension of the GSRS as the reflux dimension could explain $50-30 \%$ of the variation for most RDQ domains. Concerning the QOL, most RDQ dimensions were related to problems encountered during eating and drinking (food and drink problems) indicating that the QOL of patients was diminished due to the fact that they had less appetite, avoided eating due to their reflux symptoms and that certain food items increased their symptoms. In contrast, other studies suggested that the RDQ correlates with all QOL dimensions of the QOLRAD. However, the highest degree of correlation was found also with food and drink problems $[6,8]$. It is known that GORD patients experience the most problems with the consumption of food and drink and little for social and physical functioning $[6,24]$. The total RDQ scores and 3 of the 4 RDQ dimensions scores (GORD, heartburn and regurgitation) could be explained for $20-40 \%$ by the specific QOLRAD dimension food and drink problems.

In conclusion, the RDQ is a valid and reliable questionnaire with excellent construct validity and a good relationship to quality of life. However, in our primary care population the diagnostic value of the RDQ with a positive SAP as reference was limited. An empirical short term PPI treatment course with pre- and posttreatment evaluation might improve the RDQ's ability to discriminate between individuals with and without GORD.

\section{Acknowledgement}

We acknowledge Astrazeneca, the Netherlands, for their support.

\section{References}

1 Locke GR III, Talley NJ, Fett SL, Zinsmeister AR, Melton LJ III: Prevalence and clinical spectrum of gastroesophageal reflux: a population-based study in Olmsted County, Minnesota. Gastroenterology 1997; 112: 1448-1456.

2 Irvine EJ: Quality of life assessment in gastro-oesophageal reflux disease. Gut 2004; 53(suppl 4):35-39.

-3 Lind T, Havelund T, Carlsson R, Anker-Hansen $\mathrm{O}$, Glise H, Hernqvist $\mathrm{H}$, et al: Heartburn without oesophagitis: efficacy of omeprazole therapy and features determining therapeutic response. Scand J Gastroenterol 1997;32: 974-979.

-4 DeVault KR, Castell DO: Updated guidelines for the diagnosis and treatment of gastroesophageal reflux disease. Am J Gastroenterol 2005;100:190-200.

5 Shaw MJ, Talley NJ, Beebe TJ, Rockwood T, Carlsson R, Adlis S, et al: Initial validation of a diagnostic questionnaire for gastroesophageal reflux disease. Am J Gastroenterol 2001; 96:52-57.
-6 Kulig M, Leodolter A, Vieth M, Schulte E, Jaspersen D, Labenz J, et al: Quality of life in relation to symptoms in patients with gastrooesophageal reflux disease: an analysis based on the ProGERD initiative. Aliment Pharmacol Ther 2003;18:767-776.

7 Shaw M: Diagnostic utility of reflux disease symptoms. Gut 2004;53(suppl 4):25-27.

8 Nocon M, Kulig M, Leodolter A, Malfertheiner P, Willich SN: Validation of the Reflux Disease Questionnaire for a German population. Eur J Gastroenterol Hepatol 2005;17:229-233.

-9 Weusten BL, Roelofs JM, Akkermans LM, Van Berge-Henegouwen GP, Smout AJ: The symptom-association probability: an improved method for symptom analysis of 24 hour esophageal $\mathrm{pH}$ data. Gastroenterology 1994;107:1741-1745.

10 Svedlund J, Sjodin I, Dotevall G: GSRS: a clinical rating scale for gastrointestinal symptoms in patients with irritable bowel syndrome and peptic ulcer disease. Dig Dis Sci 1988;33:129-134.
11 Wiklund IK, Junghard O, Grace E, Talley NJ, Kamm M, Veldhuyzen van Zanten S, et al: Quality of life in reflux and dyspepsia patients: psychometric documentation of a new disease-specific questionnaire (QOLRAD). Eur J Surg Suppl 1998;583:41-49.

$>12$ Bredenoord AJ, Weusten BL, Smout AJ: Symptom association analysis in ambulatory gastro-oesophageal reflux monitoring. Gut 2005;54:1810-1817.

13 Talley NJ, Fullerton S, Junghard O, Wiklund I: Quality of life in patients with endoscopynegative heartburn: reliability and sensitivity of disease-specific instruments. Am J Gastroenterol 2001;96:1998-2004.

14 Dimenas E, Glise H, Hallerback B, Hernqvist $\mathrm{H}$, Svedlund J, Wiklund I: Well-being and gastrointestinal symptoms among patients referred to endoscopy owing to suspected duodenal ulcer. Scand J Gastroenterol 1995; 30:1046-1052.

15 Hanley JA, McNeil BJ: The meaning and use of the area under a receiver operating characteristic (ROC) curve. Radiology 1982;143: 29-36. 
16 Swets JA: Measuring the accuracy of diagnostic systems. Science 1988;240:12851293.

17 Aanen MC, Weusten BL, Numans ME, de Wit NJ, Baron A, Smout AJ: The diagnostic value of the proton pump inhibitor test for gastroesophageal reflux disease: a population-based study. Gastroenterology 2006; 130(4 suppl 2):A-162.

18 Johnsson F, Hatlebakk JG, Klintenberg AC, Roman J, Toth E, Stubberod A, et al: Oneweek esomeprazole treatment: an effective confirmatory test in patients with suspected gastroesophageal reflux disease. Scand J Gastroenterol 2003;38:354-359.
9 Numans ME, Lau J, de Wit NJ, Bonis PA: Short-term treatment with proton-pump inhibitors as a test for gastroesophageal reflux disease: a meta-analysis of diagnostic test characteristics. Ann Intern Med 2004;140: 518-527.

20 Waldum HL, Arnestad JS, Brenna E, Eide I, Syversen U, Sandvik AK: Marked increase in gastric acid secretory capacity after omeprazole treatment. Gut 1996;39:649-653.

21 Bate CM, Riley SA, Chapman RW, Durnin AT, Taylor MD: Evaluation of omeprazole as a cost-effective diagnostic test for gastro-oesophageal reflux disease. Aliment Pharmacol Ther 1999;13:59-66.
22 Juul-Hansen P, Rydning A: Endoscopy-negative reflux disease: what is the value of a proton-pump inhibitor test in everyday clinical practice? Scand J Gastroenterol 2003;38: 1200-1203.

23 Loong TW: Understanding sensitivity and specificity with the right side of the brain. BMJ 2003;327:716-719.

24 Pace F, Negrini C, Wiklund I, Rossi C, Savarino V: Quality of life in acute and maintenance treatment of non-erosive and mild erosive gastro-oesophageal reflux disease. Aliment Pharmacol Ther 2005;22:349-356. 